\title{
RE-SIGNIFICANDO LA FRONTERA: EL CASO DE LA EUROCIUDAD CHAVES-VERÍN
}

\author{
María Lois \\ Departamento de Ciencia Política y de la Administración III (Teorías y Formas Políticas y Geografía Humana). \\ Universidad Complutense de Madrid \\ mdlois@cps.ucm.es
}

\section{RESUMEN}

Las políticas de regionalización transfronteriza en las fronteras interestatales de la Unión Europea se han convertido en un emblema de su construcción geográfico-política. A través de dotación institucional y de marcos legislativos, las periferias fronterizas se han ido transformando en centros de construcción de europeidad; localizada entre el sur de Galicia y el norte de Portugal, la Eurociudad Chaves-Verín será la herramienta de exposición y discusión de prácticas en torno a la resignificación de fronteras, abordadas como imaginarios espaciales paradójicos y en constante transformación.

Palabras clave: frontera, Eurociudad Chaves-Verín, prácticas de fronterización, socialización espacial, periferias.

\section{ABSTRACT}

The European Union transborder regionalization politics have become an icon of its political geography regime. Border peripheries are getting into centrality as centers of construction of Europeanness; located in South Galicia- North Portugal, the Eurociudad Chaves-Verín will be the tool to expose and debate bordering practices, border re-signification at the EU, and as an example to advocate for a necessary border theory that may focuse in their comprehension as a multidimensional and paradoxical process.

Key words: border, Eurociudad Chaves-Verín, bordering practices, spatial socialization, peripheries.

Fecha de recepción: junio 2011.

Fecha de aceptación: diciembre 2012. 


\section{INTRODUCCIÓN}

En una conocida revisión sobre narrativas en torno a límites y fronteras, Newman y Paasi (1998) identificaban varias de las principales cuestiones en torno a las que gira la investigación en torno a las fronteras. Una de ellas, constantemente evocada en los análisis sobre procesos de globalización, es la de que las fronteras estarían en vías de desaparecer (1998: 191; ver, por ejemplo, Ohmae, 1990). Sin embargo, la producción académica en torno al tema no apunta hacia la desaparición de los límites fronterizos, sino a dinámicas bastante más complejas. En palabras de Balibar, «vivimos en una coyuntura de constante vacilación de las fronteras -tanto de su trazado como de sus funciones- que es, al mismo tiempo, una vacilación de la propia noción de frontera, que se ha convertido en particularmente equívoca» (Balibar, 1998: 216), lo que redundaría en la necesidad de desarrollar herramientas de análisis y de abordar diferentes contextos desde los que explicar sus continuos cambios.

Así, diferentes investigadores han insistido en la necesidad de adoptar una perspectiva multidimensional en el acercamiento a las fronteras (Perkmann-Sum, 2002; Paasi, 2005; Newman, 2006b; van Houtum et al., 2005: 1-14; Agnew, 2008; Kuus, 2010), y en su conceptualización no como estructuras permanentes y estáticas, sino como procesos históricamente contingentes (Newman y Paasi, 1998: 201). La frecuentemente asumida naturaleza unívoca de las fronteras ha sido reemplazada por su análisis como lugares en movimiento, como paradójicos enclaves en constante negociación. El carácter equívoco de las fronteras (Agnew, 2008: 176), y los cambiantes y contradictorios procesos en torno a su constante reproducción, se han convertido en una posibilidad de superar el acercamiento a las fronteras en términos binarios, esto es, de delimitación entre Estados; al tiempo, permite dejar de pensarlas como lugares en proceso de desaparición. Si bien las fronteras han sido proyecciones territoriales del poder infraestructural del Estado (O’Dowd, 2010), imaginarlas como las líneas fronterizas en las que se materializa el control político y social a través de la separación de espacios sólo recogería una parte de su complejidad. Las fronteras se configuran como mecanismos multidimensionales de la producción constante de estatalidad, pero en múltiples tiempos y espacios.

Estas consideraciones no están sólo en el centro del debate académico; los medios de comunicación denotan constantemente la específica materialidad histórica y significación sociocultural de las fronteras (Hiernaux y Lindón, 2006: 213). Así, el 20 de febrero de 2009, el diario La Región publicaba la siguiente noticia: «Los 18 concellos de 'Los Tres Reinos' se unen para solicitar la revitalización del tren» ${ }^{1}$. Se anunciaba una reunión prevista para el mes de marzo entre los alcaldes de la comarca de los Tres Reinos-integrada por trece municipios de Ourense (en marcados en la comarca de Verín y Conso-Frieiras), cuatro de Sanabria -Zamora y dos de Portugal-, para concretar una petición a la Xunta de Galicia y al gobierno español de la revitalización de la estación de tren de A Gudiña (provincia de Ourense). En la noticia se remarcaba la necesidad de ubicar allí una parada del AVE y de aumentar la dotación de personal, por los servicios fronterizos que podría prestar esta localización.

1 La Región, 20-02-2009 [Edición digital], <http://www.laregion.es/noticia/82487/gudi\%C3\%B1a/concellos/tres/reinos/revitalizaci\%C3\%B3n/tren/> [ consultado el 22 de septiembre de 2009] 
En la primavera de 2010, los alcaldes de cinco municipios portugueses de la frontera, en torno a Valença do Minho, reclamaban que sus más de 61.000 habitantes pudieran ser atendidos en el servicio sanitario de Urgencias del hospital de Vigo, situado a unos quince minutos por carretera, más cercano que el oficialmente designado a tal efecto por el gobierno portugués, localizado en Viana do Castelo $^{2}$. La petición se hizo en medio de una protesta vecinal en la que la bandera española se convertía en símbolo de agradecimiento de los portugueses al alcalde del fronterizo municipio de Tui, que ofrecía su servicio de Urgencias como posible solución al descontento.

El referente de ambas noticias es un imaginario común: el de la reformulación de los límites para la acción social y política. La alusión a la comarca de los Tres Reinos como escenario de gestión o la reivindicación del uso de un servicio público localizado al otro lado de la línea fronteriza llaman la atención sobre las constantes transformaciones de las geografías oficiales, mostrando que las nociones de límites y de fronteras tienen una especificidad cultural e histórica, y que son procesos de carácter multidimensional. Transforman las percepciones y definiciones de lo político, lo social, lo cultural, lo personal (Cairo, 2001: 35), y establecen y marcan referentes, contextos geohistóricos de representación del espacio y el tiempo colectivos, y por tanto, de producción y reproducción del orden social. Estos sistemas de reproducción son, metafóricamente hablando, creados por la economía mundo moderna (Smith et al. 1984: 13), y afectarían no solo a las prácticas materiales, sino también a la «comunidad de destino», donde vivimos nuestras vidas cotidianamente y desde donde construimos los horizontes de las prácticas y representaciones sociopolíticas. Las formas en las que se crean y re-crean esas comunidades están en cambio constante. La socialización espacial, entendida como proceso a través del que los actores individuales y las colectividades son socializados como miembros de entidades territorialmente delimitadas y adoptan formas específicas de pensamiento y acción (Paasi, 2007:15; 1996: 54), está en permanente construcción y reconstrucción. Por tanto, el orden socioterritorial, también.

En este trabajo el acercamiento a la frontera se producirá desde su comprensión como un proceso inacabado, desde lo que se ha denominado prácticas de fronterización (bordering practices). Si las fronteras crecieron como parte de los Estados y de su creación, las identificaciones políticas se construyen a través de prácticas de fronterización (Kuus, 2010: 671-672), entendidas como «una amplia gama de procesos transformativos y afectivos en los cuales los órdenes y desórdenes sociales y espaciales son constantemente reelaborados» (Woodward y Jones, 2005: 239). Re-pensar las fronteras a través de las prácticas de fronterización supone entender esas prácticas como algo implícito en la construcción de esas fronteras, no analizables como desarrollos incompletos o acabados, «sino en constante proceso de materialización» (Prokkola, 2008b: 15).

Desde ese punto de partida, el objetivo de este artículo es presentar la Eurociudad ChavesVerín, como ejemplo de desarrollo de las políticas entre límites fronterizos interestatales dentro de la Unión Europea, y de los esquemas de socialización espacial que operan en torno a ellas. Para ello, se expondrá a grandes rasgos la retórica de las políticas de regionalización transfronteriza de la UE, así como sus principales líneas de acción. A continuación, se

2 El País, 05-04-2010, [Edición digital], <http://www.elpais.com/articulo/internacional/Banderas/espanolas/ protestar/recortes/sanitarios/Portugal/elpepuint/20100405elpepuint_12/Tes $>$ [consultado el 6 de mayo de 2010] 
describirán los ejes de creación y funcionamiento de la Eurociudad, así como sus proyectos base, seguida de una discusión en términos de su incidencia y de las implicaciones para múltiples geografías políticas transfronterizas. El escrito se cierra con unas reflexiones en torno a la performatividad de las fronteras, a partir de las prácticas de fronterización en las fronteras internas de la UE y de su materialización en las geografías locales.

\section{LA UNIÓN EUROPEA Y LAS FRONTERAS INTERESTATALES: REGIONES, COOPERACIÓN TRANSFRONTERIZA, TURISMO}

El desarrollo de la UE y los procesos ligados a lo que se ha llamado integración ha incidido de forma clara en los procesos de asociación e identificación de límites y fronteras. No sólo por las dinámicas que conforma en cuanto a la demarcación constantemente cambiante de sus propios límites, sino por la incidencia de sus políticas en la que conocemos como configuración clásica de las fronteras como límites entre Estados-nación. Así, la imaginación espacial ligada a la Unión Europea es la de la Europa de las regiones, donde estas funcionarían como un conjunto de entidades funcionales que conectarían y mejorarían las competividad de las regiones en diferentes escalas. Este proceso de europeización se materializa en «la introducción de nuevos procesos y estructuras institucionales que funcionan en nuevas escalas y transgreden las fronteras estatales, creando nuevas posibilidades de acción» (Jensen y Richardson, 2004: 24). La política regional de la Unión Europea se basa en crear regiones capaces de desempeñar plenamente su papel en favor de un mayor crecimiento y competitividad e intercambiar al mismo tiempo ideas y buenas prácticas. Este es precisamente el objetivo de la iniciativa «Las regiones, por el cambio económico» ${ }^{3}$ : la política regional en su conjunto coincide con las prioridades que se fija la Unión Europea en favor del crecimiento y el empleo, tal y como se refleja en la Estrategia de Lisboa.

Dentro de ese proceso, la regionalización transfronteriza se habría convertido en un laboratorio a través del cual se ha medido el éxito del proceso de integración europea (Knippenberg, 2004). Si conceptualizamos una región transfronteriza como una unidad territorial que contiene unidades subnacionales de uno o más Estados (Perkmann y Sum, 2002:3), el proyecto de regionalización de la Unión Europea sería un proceso que implicaría, al menos teóricamente, tanto a los actores regionales y locales (institucionales y no institucionales; públicos y privados) en estrategias de desarrollo, a través de la promoción de formas de gestión donde el Estado no sería el principal agente de regulación política.

En términos de prácticas institucionales, el 75\% de las regulaciones de la UE son implementadas a nivel regional (Evers, 2006: 81, citado en Lambregts et al., 2008: 46), y alrededor de un tercio del presupuesto comunitario se destina al desarrollo regional. Instrumentos como las iniciativas INTERREG o las Redes Transeuropeas suponen el marco regulador de la construcción del desarrollo de regiones transfronterizas; de hecho, el territorio de la Unión

3 <http://ec.europa.eu/regional_policy/cooperation/interregional/ecochange/doc/comm_es_acte.pdf> [consultado el 25 de febrero de 2009]; <http://ec.europa.eu/regional_policy;http://ec.europa.eu/regional_policy/ faq/q7/index_es.htm>; <http://europa.eu/scadplus/glossary/lisbon_strategy_es.htm>, [consultado el 25 de febrero de 2009]; «Informe estratégico sobre la estrategia de Lisboa renovada para el crecimiento y el empleo: lanzamiento de un nuevo ciclo (2008-2010), parte I», Comunicación de la Comisión al Consejo, de 11 de diciembre de 2007, [COM(2007) 803 final - no publicado en el Diario Oficial].) 
Europea suele presentarse como $\mathrm{el}$ territorio de fronteras por excelencia: «las regiones fronterizas representan el 40\% del territorio de la Unión Europea y un 25\% de su población [...] Las regiones situadas enteramente a lo largo de las fronteras internas de la Unión, esto es, las fronteras entre estados miembros, forman un grupo heterogéneo que supone el $27 \%$ del territorio de la Unión y el 18\% de su población» (EC 2002). Tras las sucesivas ampliaciones, «el aumento del suelo fronterizo comunitario y su extensión implica que el valor añadido de la cooperación transfronteriza (...) debe ser incrementado» (EC 2006/1083). En ese sentido, los programas de trabajo de la Unión Europea para resignificar las fronteras entre los Estados miembros se basan en un modelo de desarrollo cuyo eje fundamental sería el turismo. Especialmente desde la firma del Tratado de Maastricht, en 1992, el turismo ha pasado a ser oficialmente reconocido como uno de los ejes fundamentales del desarrollo promovido por la UE: «La infraestructura creada para el turismo contribuye al desarrollo local, y se crean o mantienen puestos de trabajo incluso en zonas en declive industrial o rural, o que están en proceso de regeneración urbana. La necesidad de mejorar el atractivo de las regiones sirve de incentivo para que un número creciente de destinos y partes interesadas promuevan prácticas y políticas más sostenibles y positivas respecto al medio ambiente» ${ }^{4}$. El turismo se representa como futura actividad económica y motor de desarrollo en las periferias rurales de Europa, entre las que se encuentras las zonas fronterizas de los diferentes Estados. Así, el desarrollo del turismo estaría vinculado con la promoción de una conciencia regional y, al tiempo, refleja la ambición de promover la integración a través de las fronteras internas de la Unión (Prokkola, 2007: 124). Este modelo de desarrollo, presente no sólo en el mencionado INTERREG, sino en programas como el LEADER, la Carta Europea del Turismo Sostenible o la Agenda 21, presenta características específicas: «El turismo sostenible desempeña un papel esencial en la preservación y la rehabilitación del patrimonio cultural y natural en un número creciente de ámbitos, que abarcan desde el arte a la gastronomía local, pasando por los oficios o la preservación de la biodiversidad» ${ }^{5}$.

Las iniciativas de la Unión Europea, entonces, suponen una fuente de financiación para las actividades fronterizas, con la intención de «promover redes transfronterizas e identidad regional», incluyendo un subprograma para iniciativas turísticas y culturales ${ }^{6}$. Las regiones periféricas se integrarían así en redes institucionales y centros de toma de decisiones; las prácticas y los discursos de desarrollo que afectan a estos ámbitos «vinculan el destino turístico a estructuras regionales y económicas más amplias, y, finalmente, a la economía mundial y la circulación de capital y de cultura. Al mismo tiempo, sirven como una herramienta y un medio institucional para el desarrollo y la construcción de la idea de una región» (Saarinen, 2004:169). Estos lugares de desarrollo se convertirían en lugares turismo enclávico -una forma específica de reproducción de actividades económicas- pero también una forma de re-crear las representaciones de los contextos locales, en permanente procesos de encuentro con las expectativas turísticas. La puesta en marcha de planes específicos de actuación en

4 <http://europa.eu/legislation_summaries/enterprise/industry/n26107_es.html>. [consultado el 25 de febrero de 2009]

$5<$ http://europa.eu/legislation_summaries/enterprise/industry/n26107_es.html>. [consultado el 25 de febrero de 2009]

6 INTERREG IIIA Nord- Fondo Europeo de Desarrollo Regional. < www.interregnord.com $>$ [consultado el 26 de febrero de 2009] 
las fronteras interestatales abre un proceso de resignificación de las prácticas y los discursos sobre los límites, cómo se producen y cómo se representan y recrean cotidianamente.

De hecho, un buen número de investigaciones sobre prácticas de cooperación transfronteriza (Scott, 2000; Kaplan y Häkli, 2002; Perkmann, 2003; Strüver, 2005; Prokkola, 2008b; Lois, 2009; 2010) muestran como ésta adopta formas muy diferentes e inciden en estas diversidades como especialmente importantes en la organización socioespacial europea. A la hora de trabajar con fronteras interestatales en la Unión Europea, debemos incorporarlas no solo como delimitaciones de comunidades políticas e identificaciones territoriales, sino también como espacios de conexión, interdependencia y cooperación interregional (Perkmann y Sum, 2002; Newman, 2006), tal y como se plantea en la retórica de la UE.

Este contexto será el marco de interpretación de las prácticas de regionalización y cooperación transfronteriza en el tramo norte de la Raia Si bien el estudio de los modelos y posibilidades de la cooperación en esta frontera son una línea de investigación relativamente consolidada $^{7}$, la aparición de la forma Eurociudad, y, específicamente, de la Eurociudad Chaves-Verín, será el desde el que abordar las paradojas ligadas a la construcción institucional de espacios transfronterizos.

\section{UN LABORATORIO TRANSFRONTERIZO: LA EUROCIUDAD CHAVES-VERÍN}

En el documento de ejecución del Programa Operativo de Cooperación Transfronteriza España-Portugal (POCTEP) para Galicia-Norte de Portugal (2001-2013), se define como eje de trabajo fundamental «el fomento de la cooperación e integración social e institucional, a través del refuerzo de la cooperación entre entidades de la administración pública regional, agentes económicos,sociales y culturales de los dos lados de la frontera; de la promoción de una mayor interacción cultural y social entre las poblaciones rayanas, y del desarrollo de servicios comunes, a través de la utilización compartida y/o en red de equipamientos sociales, culturales y de ocio (POCTEP, 2009: 55).

En el marco de esta política comunitaria, y a partir del impulso de los gobiernos municipales de la ciudad de Chaves en el norte de Portugal, y Verín, en el sur de Galicia, se creó, en diciembre de 2007, la Eurociudad Chaves-Verín, en el valle del río Támega ${ }^{8}$. El proyecto, que presentaba como socios al municipio de Verín, al municipio de Chaves, al Eixo Atlántico del Noroeste Peninsular, a la Dirección Xeral de Xuventude e Solidariedade de la Xunta de Galicia, al Instituto Português da Juventude (IPJ), y a la Deputación de Ourense, comenzaba con un coste de 1.333.578,20 euros, y una cofinanciación solicitada a los Fondos Europeos de Desarrollo Regional (FEDER) de un 75\% (Eurociudad Chaves-Verín: la Eurociudad del Agua, 2011: 3). La Eurociudad se conformaba así como una estructura sin un marco jurídico específico y definido, pero siguiendo las tendencias de cooperación de segunda generación recomendadas por las iniciativas de la Unión Europea.

7 Ver, por ejemplo, Cabero, Campesino y López Trigal, 1995-1996; López Trigal, Lois González y Guichard, 1997; Márquez, 1997; López Trigal, 2000; Guichard, López Trigal y Marrou, 2000; o Lois González y Plaza, 2003.

8 Esta no es la primera experiencia en ese sentido en las fronteras ibéricas; así la Eurociudad Bayona-San Sebastián, en la frontera entre el País Vasco y Francia comenzó a gestarse en 1993 (Lozano Valencia, 2007) 
Figura 1

LOCALIZACIÓN DE LA EUROCIUDAD CHAVES-VERÍN

LOCALIZACIÓN EUROCIUDAD, VERÍN (España) - CHAVES (Portugal)

EUROPA

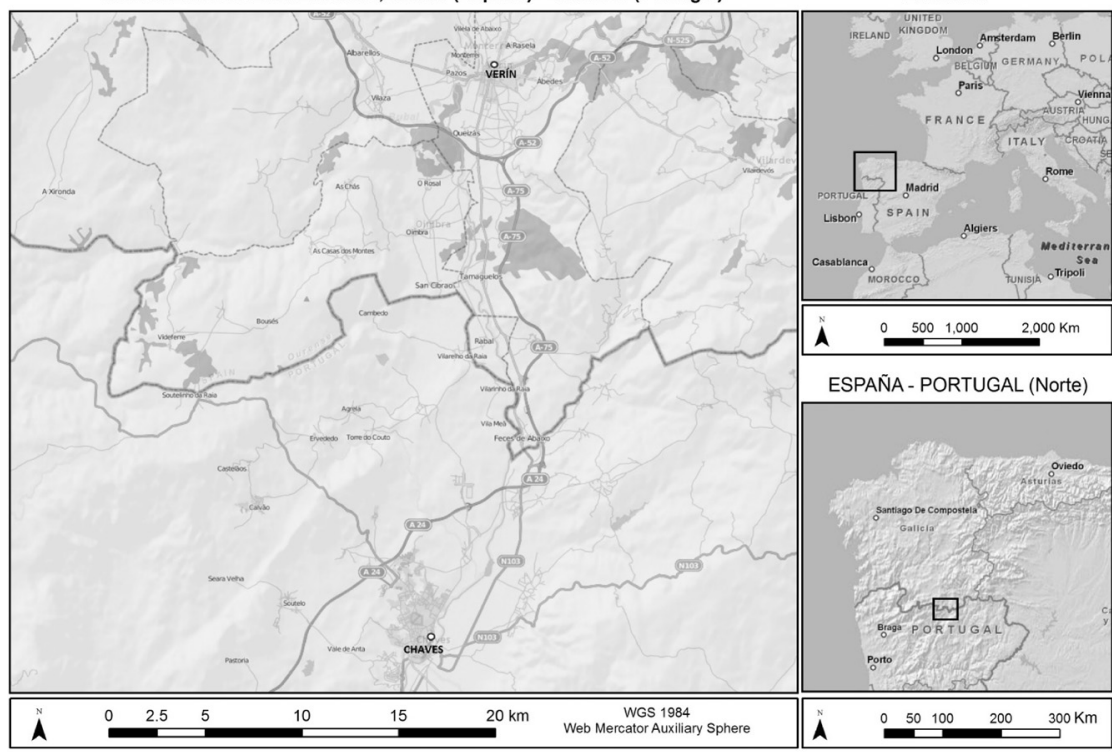

Fuente: Openstreet Maps y ESRI. Elaboración: J. Díaz Pacheco

En disonancia con otras localidades del área fronteriza de la Raia Seca, ambas localidades presentan un incremento de población desde los años 90, en un entorno regional de éxodo de la población joven y de envejecimiento poblacional generalizado, así como una fuerte terciarización en los sectores de actividad económica.

Tabla 1

DATOS SOCIODEMOGRÁFICOS DE CHAVES Y VERÍN

\begin{tabular}{|l|l|l|}
\hline & \multicolumn{1}{|c|}{ Chaves(Portugal) } & \multicolumn{1}{c|}{ Verín(España) } \\
\hline Extensión & $591,2 \mathrm{~km}^{2}$ & $94,1 \mathrm{~km}^{2}$ \\
\hline Población & $43.667 \mathrm{hab}$. & $14.391 \mathrm{hab}$. \\
\hline Densidad de población & $74,2 / \mathrm{km}^{2}-51$ freguesías & \\
\hline Actividades Económicas & Comercio, turismo & $\begin{array}{l}\text { Producción y distribución vinícola, } \\
\text { producción y embotellamiento de } \\
\text { agua, hostelería, comercio }\end{array}$ \\
\hline
\end{tabular}

Fuente: Dossier Eurocidade, 2009; Instituto Nacional de Estadística (INE), Instituto Galego de Estadística (IGE), Instituto Nacional de Estatística de Portugal.

Elaboración propia.

9 Las freguesías en Portugal y las parroquias en Galicia son los términos utilizados para designar una unidad administrativa local. En Portugal tienen alguna competencia administrativa y electoral, pero en Galicia son subdivisiones de los municipios, $\mathrm{y}$, aunque como entidades colectivas no tengan valor legal, son constantemente utilizadas como referentes tanto en textos legales como en medios de comunicación, gestión pública, celebraciones festivas etc. 
Tanto Chaves como Verín funcionan como núcleos de empleo y comercio para las áreas que las rodean, de carácter rural, y presentan una concentración de población superior al 50\% respecto a la totalidad de parroquias que integran ambos municipios; en el caso de Verín, el municipio es la segunda unidad de población en términos censales de la provincia de Ourense, por detrás de la capital. En el caso de Chaves, pese a ser el mayor de los municipios de la Región del Alto Trás-os-Montes, la concentración de población va en paralelo a un aumento del desempleo, al igual que en Verín ${ }^{10}$.

La primera línea de acción de la Eurociudad fue la creación, en 2007, del Centro Transfronterizo de Chaves- Instituto de la Droga y de la Tóxicodependencia, actuación conjunta entre la Delegación Regional del Norte del Instituto de Tóxicodependencia de Portugal (IDT) y su homóloga gallega, el Servicio Galego de Saúde (SERGAS). El Centro Transfronterizo de Chaves, más allá de abarcar la región del Alto Trás-os-Montes portuguesa, sirve también a los usuarios de la provincia de Ourense y, especialmente, de la Mancomunidade de VerínMonterrei, constituyéndose como el primer servicio transfronterizo en la Eurociudad. Fue desarrollado con el auspicio del programa INTERREG III-A, siendo el acceso a de servicios de salud en hábitats dispersos, como se mencionaba más arriba, una de las demandas con más apoyo social.

Más allá de este primer servicio, los ejes de acción de la Eurociudad se han ido concretando en una matriz operativa, dividida en tres áreas de actividades. Por un lado, las acciones diseñadas en torno al Desarrollo de la Eurociudadanía. El objetivo es «fomentar el sentido de pertenencia a una misma unidad territorial» (Eurociudad Chaves-Verín: la Eurociudad del Agua 2011:11). Para ello, se gestiona una agenda cultural común, a través de la que se programan diferentes actividades (torneos de fútbol, excursiones de senderismo, exposiciones de artistas locales de ambos lados, concursos de creación de mascota, etc.). Además, en este eje se proyecta el desarrollo de un sistema de transporte colectivo de la Eurociudad, la rehabilitación del antiguo edificio de aduanas de Feces da Cima (Verín) para crear una Ventanilla Única, una Oficina de Juventud y un local de ensayos transfronterizos (Eurociudad ChavesVerín: la Eurociudad del Agua 2011:11), y la creación de una tarjeta de Eurociudadano, como documento de pertenencia y de posibilidad de libre circulación y del uso de equipamientos públicos en toda la Eurociudad.

Una segunda línea de trabajo sería la desarrollada en torno a la Dinamización Económica. Así, la Eurociudad patrocina paquetes de turismo integral en torno a los servicios termales del río Támega; promueve la creación de un área y un directorio comercial común, llamado Frontera Comercial Abierta, de un parque logístico desde el que ordenar el intercambio de mercancías entre ambos países ${ }^{11}$, y de un sistema de apoyo a las iniciativas empresariales, tanto para la creación como para la consolidación de emprendedores en ambos lados (Dossier Eurociudad, 2009:3-10).

10 La cifra de desempleo en Chaves es de un 17\%, según los datos Instituto de Emprego e Formaçao Professional de Portugal (www.iefp.pt. [consultado el 16 de marzo de 2011]); en el caso de Verín, el desempleo afectaría a un 18,5\% de la población, según datos del Ministerio de Trabajo e Inmigración, (<https://www.redtrabaja. es/es/redtrabaja/static/Redirect.do?page=statsEmpleo $>$ [consultado el 16 de marzo de 2011])

11 Varios de los textos marco de la Eurociudad, así como una información más amplia sobre estas y otras líneas de acción se encuentra en la web institucional(< http://es.eurocidadechavesverin.eu/>) 
Y, por último, el eje de trabajo referido como Desarrollo Sustentable. En esa área, se trataría de impulsar una Eurociudad Ecológica del Tàmega, a través de la valorización del río como símbolo de los valores naturales, históricos y culturales de la zona (Eurociudad Chaves-Verín: la Eurociudad del Agua, 2011:13). Otra de las actuaciones previstas englobaría un plan de ordenación conjunta del territorio de la Eurociudad.

Para los años 2011-2012, la Eurociudad ha presentado cuatro candidaturas a la convocatoria del POCTEP, con la intención de crear un Centro de Formación Turístico-Termal y de Investigación del Agua; una Ciclovía en los márgenes del río que una las dos municipalidades, o de consolidar la presencia de la Eurociudad en la Red de Entidades Transfronterizas (RIET), a través de la creación de un Centro de Documentación Transfronteriza con sede en Chaves-Verín.

\section{INTERPRETANDO EL LABORATORIO: LA IMAGINACIÓN TRANSFRONTERIZA}

Los proyectos de regionalización transfronteriza en el marco de la Unión Europea suponen un campo experimental, por su vinculación a un cambio en la significación de la frontera. De zona periférica para los Estados, se convierten en nodos receptores y transmisores de pautas de inversión de significados histórica y socialmente construidos, en términos espaciales. Esa reinversión simbólica se convierte en un proceso cotidiano de construcción de referentes y de negociación constante en torno a las connotaciones y representaciones de la zona transfronteriza como área y espacio unificado y ahora institucionalizado, que despliega cartografías oficiales paralelas a sus dimensiones de emplazamientos fronterizos. Así, las prácticas diseñadas desde y para la Eurociudad la significan como espacio social, cultural, político e institucional que abre, en términos de referentes geográficos, los horizontes de representación e imaginación geográfica de la comunidad. La Eurociudad se ha ido configurando como agente de socialización espacial cotidiano en los municipios de Chaves y Verín, aunque con desigual incidencia ${ }^{12}$. Sin embargo, la continua organización de actividades culturales y lúdicas, así como la presencia en ciclos festivos ha ido fomentando una visibilidad del proyecto a nivel local. Actividades como la Agenda Cultural conjunta, los programas de Termalismo Social (tratamientos termales prolongados y conjuntos para mayores de 50 años) y, sobre todo, a través de la organización de eventos en torno a la Eurociudad en centros educativos y en ciclos festivos. En ese sentido, actividades como el Día del Niño conjunto, la celebración de un concurso para la creación de Chavín, la Mascota de la Eurociudad, las llamadas «Convivencias con las Lenguas» (actividades lúdico-formativas que fomenten el aprendizaje del castellano y el portugués durante las vacaciones estivales en niños entre 4 y 14 años), la adaptación juegos como el Parchís o el Trivial a la historia y la cultura de la

12 En una valoración sobre la cuestión, tanto Ana Ladeiras, impulsora del proyecto y coordinadora técnica de la Eurociudad en Chaves, como Pablo Rivero, coordinador del Gabinete Técnico de la Eurociudad en Verín, o el secretario general del Eixo Atlántico, Xoaquín Váquez Mao, afirman que existe una acogida diferencial entre ambos municipios; el de Chaves siempre habría sido más entusiasta, con un apoyo unánime, en términos políticos y sociales, de la iniciativa; en el caso de Verín, coinciden en señalar un mayor escepticismo entre la población, así como un uso de la Eurociudad como elemento de confrontación partidista (entrevista semiestructurada con Ana Ladeiras, realizada 28 de julio de 2009; entrevista semiestructurada con Pablo Rivera, realizada el 10 de febrero de 2011; y La Región, 05.01.2009 < http://www.laregion.es> [consultado el 3 de marzo de 2011].) 
Figura 2

MATERIAL DISTRIBUIDO POR LA EUROCIUDAD EN ACTIVIDADES INFANTILES

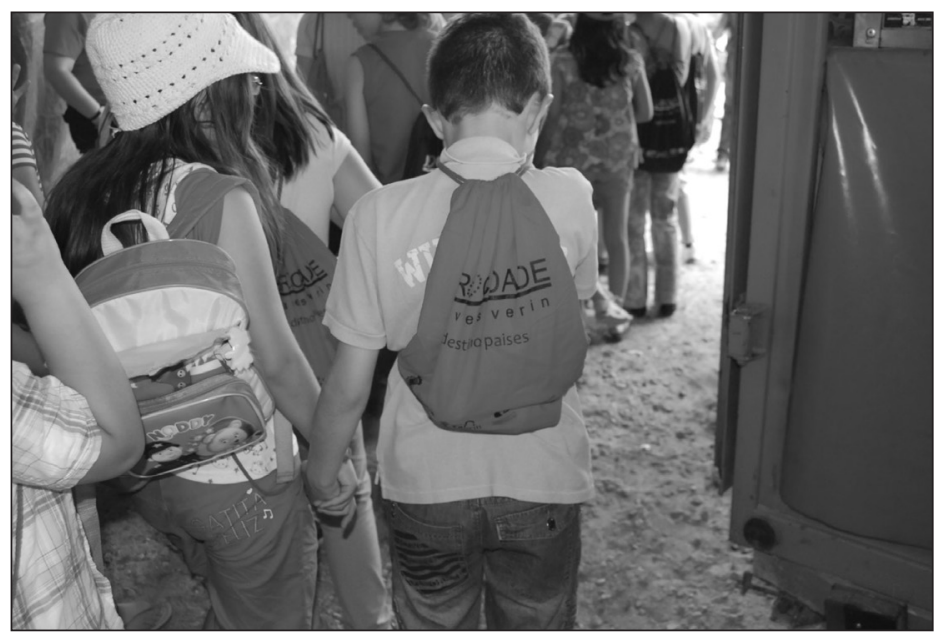

Fuente: fotografía cedida por la Coordinación del Gabinete Técnico de la Eurociudad Chaves-Verín.

Figura 3

CARTEL QUE ANUNCIA UNA RECOGIDA DE ALIMENTOS PROMOVIDA POR LA EUROCIUDAD, DENTRO DE LAS ACTIVIDADES NAVIDEÑAS

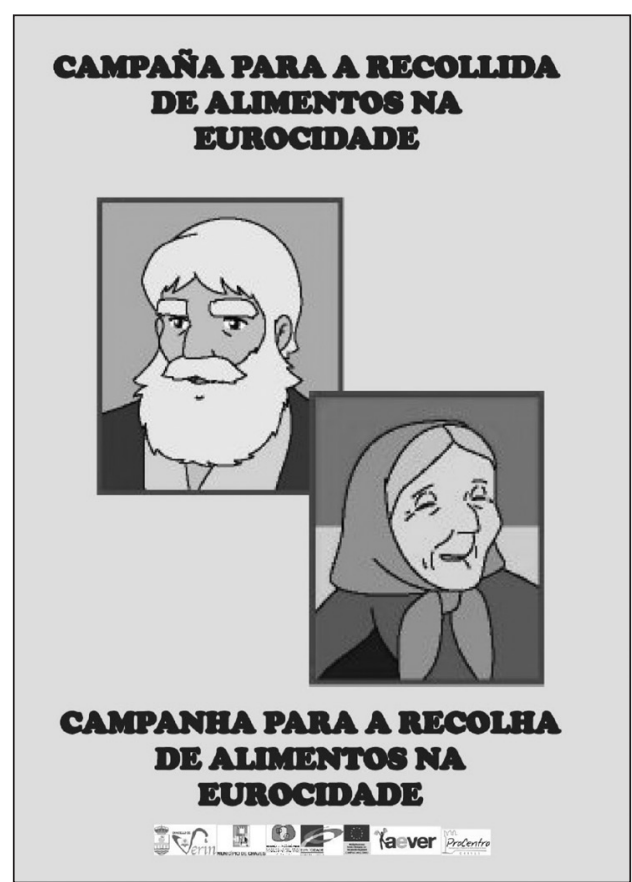

Fuente: http://es.eurocidadechavesverin.eu/actividades/navidad-en-la-eurocidade-2010-2011 [consultado el 23 de marzo de 2011] 
Eurociudad (Eurociudad Chaves-Verín: la Eurociudad del Agua, 2011:12), o la asistencia de unos dos mil niños ${ }^{13}$ de ambas localidades a la II Semana de Educación Viaria sin duda habilitan formas de socialización espacial en entornos educativos y lúdicos que remitirían a una imaginación espacial conjunta. Igualmente, en las navidades de 2010, se organizaron actividades específicas para niños desde la Eurociudad, como campañas conjuntas de recogida de juguetes y alimentos o ludotecas ${ }^{14}$.

Además de este público implicado en actividades con un horizonte transfronterizo, varias de las intervenciones más emblemáticas de la Eurociudad afectan directamente a la re-construcción de la frontera como un marcador simbólico con dimensiones espaciales significativamente invertidas respecto a su conformación clásica; una de ellas es la creación de una tarjeta de acreditación de la Eurociudadanía. Dicha tarjeta, en proceso de divulgación pero ya presentada al público ${ }^{15}$, sería un documento que permitiría utilizar los equipamientos públicos de ambos municipios, así como ciertas ventajas en el uso del sector privado. No exenta de problemas de gestión estatal diferenciada, ya que los sistemas administrativos de controles de acceso a los equipamientos son incompatibles, la tarjeta evoca en un documento oficial de identificación un territorio diferenciado, con acceso diferenciales en función de su pertenencia a una entidad especialmente definida en base a su localización fronteriza, y en paralelo a otras formas de identificación territorial legalmente reconocidas. El horizonte simbólico de esta tarjeta remite a un imaginario espacial en construcción, y en proceso de socialización. En esta línea, el proyecto de crear una Zona Franca Social también ahondaría en esa consolidación de un escenario territorialmente diferenciado, que contestaría los emplazamientos tradicionales. Así, ahondando en la línea de fortalecer el proyecto a través de prácticas que contengan al nuevo escenario espacial como referencia cotidiana, el propósito de crear esta Zona Franca sería consolidar un área con acceso a servicios comunes en términos culturales (creación de una Escuela de Letras, programación de actividades conjuntas); de educación (módulos de enseñanza secundaria comunes y conservatorio), y salud (acceso a la prestaciones hospitalarias por parte de los vecinos de ambas localidades) ${ }^{16}$.

Sin embargo, la acción que proyectaría, de alguna manera, la reconfiguración de la frontera como límite interestatal y que la proyectarían como icono espacial de nuevos valores sería la rehabilitación de edificios representativos con la intención de resignificarlos en un nuevo escenario transfronterizo. En el lado español, el edificio de aduanas está localizado en la parroquia de Feces de Abaixo (Verín), y, tras ser cedido al Ayuntamiento de Verín, está en fase de remodelación para su futura utilización. En este edificio, se localizarán, demás del Gabinete Técnico de la Eurociudad, una Ventanilla Única, una Oficina Transfronteriza de Juventud, promovida por la Xunta de Galicia y el Instituto da Juventude portugués, y un local de Ensayos. La rehabilitación está financiada en un 75\% por los fondos FEDER, y por

13 Estimación hecha por el coordinador del Gabinete de Apoyo Técnico de la Eurociudad en Verín, en entrevista semiestructurada realizada el 10 de febrero de 2011.

$14<$ <ttp://es.eurocidadechavesverin.eu/actividades/navidad-en-la-eurocidade-2010-2011>, [consultado el 3 de marzo de 2011]

15 La Región,05-01-2011, <http://www.laregion.es/noticia/138955/carne/eurociudadano/permite/compartir/ servicios/verin/chaves/>. [consultado el 3 de marzo de 2011]

16 La Región, 16.07.2008, <http://www.laregion.es/noticia/62877/Ver\%EDn/Chaves/Eurociudad/> [consultado el 3 de febrero de 2009] 
el POCTEP. La localización de infraestructuras socioculturales dirigidas a los usuarios de ambos lados, y marca una conversión simbólica clave. La representación del límite fronterizo y del registro y fiscalización de las actividades de tráfico entre dos Estados se convierte en el distrito centro de la nueva marca, ahora pensada en términos de una misma ciudad, pero con dos barrios (Barrio de Arriba y Barrio de Abajo) ${ }^{17}$. La periferia fronteriza se convierte en un distintivo de demarcación espacial que remite a horizontes espaciales con contenidos propios, desde los que se enmarcan y producen prácticas de socialización espacial. Esa periferia fronteriza se va constituyendo en un destino propio, con dimensiones espaciales diferenciadas, que trascienden su propia delimitación.

La distinción de la Eurociudad como «Mejor Destino Turístico de Futuro» otorgado por la revista «Viajes y Turismo» en el año 2009 señala su configuración como destino final y no sólo no como lugar de paso -en términos turísticos-, con una oferta propia. Y no sólo en términos de paquetes vacacionales, sino también en términos de lo que se podría denominar turismo académico. La Eurociudad se ha convertido en destino de reuniones y visitas encaminadas a conocer y promover la Eurociudad como destino final. Ha recibido visitas de estudiantes de las universidades de Tours (Francia), de varias de las universidades portuguesas (Lisboa, Trás-os-Montes), y ha sido sede, en marzo de 2009, de un Seminario sobre «Conurbaciones transfronterizas: potenciación de la gobernanza interna», enmarcado en el programa europeo URBACT, o de la última reunión de la Red Ibérica de Entidades Transfronterizas (RIET), en febrero de 2011. Igualmente, una delegación del gobierno de Brasil visitó el proyecto en septiembre de 2010, en el marco del Memorando de Entendimiento entre la Comisión Europea y el Ministerio de Integración de Brasil. Además de recibir la tarjeta de Eurociudadanos, la Comisión, integrada entre otras personas, por representantes de las Meso-Regiones de Alto Simoneo, Vale do Río Acre y de la Gran Fronteira Mercosur intercambió experiencias en proyectos de alcance transfronterizo ${ }^{18}$.

Las estrategias de construcción de la frontera como recurso traspasarían las referencias espaciales más cercanas para convertirse en referente de gestión de nuevos imaginarios territoriales. Las referencias del proyecto transmiten esquemas de socialización espacial que de alguna manera van re-creando un escenario de acción y participación que podría configurarse como patrón de escenificación de la comunidad de destino, - - en este caso, la de Chaves-Verín-, como referente de actuación política y social que va creando un escenario no sólo sociocultural, sino como un espacio de proyección político-institucional en plena negociación más allá de sus fronteras locales. En ese sentido, los cambios de prioridad en la imaginación geográfica de referencia en las proyecciones políticas a escala autonómica han marcado los últimos dos años del desarrollo del proyecto. De hecho, la cooperación transfronteriza entre Galicia y el norte de Portugal, y en especial, la Eurociudad Chaves-Verín se ha conformado como un proyecto de imaginación transfronteriza, revelándose como un escenario institucional patrimonializable en términos de imaginación política.

17 La Región, 16.07.2008, <http://www.laregion.es/noticia/62877/Ver\%EDn/Chaves/Eurociudad/> [consultado el 3 de febrero de 2009], y entrevista al coordinador del Gabinete de Apoyo Técnico a la Eurociudad, 10 de febrero de 2011.

18 Web institucional de la Red Ibérica de Entidades Transfronterizas (RIET), <http://www.rietiberica.eu/ riet/index.php?option=com_content $\&$ view=article \&id=352\%3Auna-delegacion-del-gobierno-brasileno-visita-laeurociudad-chaves-verin\&catid=98\%3Aasociacionesredibericda\&lang=es $>$ [consultado el 3 de marzo de 2010] 
A escala autonómica, por ejemplo, el Grupo de Trabajo de la Eurociudad Chaves-Verín vincula el proyecto no sólo con la Xunta de Galicia, sino también con el Eixo Atlántico, asociación transfronteriza de derecho privado, integrada por los municipios urbanos de la Eurorregión Galicia-Norte de Portuga ${ }^{19}$. La centralidad que adquiere la Eurociudad, en el marco de una proyección de espacios de gestión política transfronterizos, se ha ido convirtiendo en una variable de resignificación de los límites de los posibles escenarios políticos autonómicos. La imaginación política transfronteriza se convierte así en un recurso de referencia para construir una visión de la frontera como lugar de intervención esencial.

A escala estatal, el proyecto de la Eurociudad ha transferido una connotación espacial diferencial a los procesos políticos; en ese sentido, la espacialidad específica del proyecto desempeñaría un papel todavía secundario en el panorama de escenarios institucionales; no obstante, en una nota de prensa previa a una reunión con entre el presidente de la Xunta de Galicia y el ministro de Fomento, se establecía la importancia de la Eurociudad como espacio vertebrador de las infraestructuras y redes de transporte viario de la península ${ }^{20}$. Aunque no se ha configurado como tema prioritario, sí que la referencia a la Eurociudad se plasma como referente en el momento de construir una zona transfronteriza con identidad propia frente a los gobiernos estatales; así, ante la futura implantación en abril de 2011 de un peaje en la autovía portuguesa A-24, que une Chaves con Viseu y que enlaza con la A-75, que une Verín con Feces de Abaixo en el lado español, los alcaldes de ambos municipios acordaron elevar una protesta al Ministerio de Obras Públicas de Portugal. La alternativa sería, según João Batista, presidente de la Cámara Municipal de Chaves, que las cabinas de cobro se instalaran lejos de Chaves, para «que no afecten al proyecto de la Eurociudad» ${ }^{21}$. La imaginación geográfica transfronteriza y la circulación por la frontera se superpone a otros proyectos de mayor alcance territorial; la vertebración estatal se superpone a la regionalización promovida por la UE, e impulsada por actores locales y regionales. No obstante, la concreción del imaginario transfronterizo de la Eurociudad en gestión de políticas públicas se dificulta notablemente a la hora de desarrollar políticas conjuntas en torno a competencias no transferidas desde las escalas estatales; la generalización de la tarjeta de Eurociudadanía, la puesta en marcha de una red de transporte público colectivo, o la consolidación de asistencia sanitaria conjunta muestran los obstáculos para desarrollar experiencias que vayan más allá de las actividades culturales o deportivas. Sin embargo, en un incipiente marco de configuración de posibilidades de acción conjunta, la constitución de la Eurociudad como Agrupación Europea de Cooperación Territorial (AECT) aprobada por ambos municipios en septiembre de 2010 dotaría de personalidad jurídica, aunque sin competencias en política exterior, en administración de Justicia o poderes policiales ${ }^{22}$. Esta ampliación competencial recoloca, de algún modo, la entidad propia del proyecto, que, una vez aprobada por las respectivas autori-

19 El Eixo Atlántico se funda en 1992, con base en el Convenio Marco sobre Cooperación Transfronteriza del Diario Oficial de las Comunidades Europeas (D.O.C.E.) del 16 de octubre de 1990, y funcionaría sobre la base de consolidarse como lobby de Galicia y Portugal en la Unión Europea (ver www.eixoatlantico.com)

20 Galicia Digital, 06.02.2009, <http://www.galiciadigital.com/noticia.4383.php>[ consultado el 3 de marzo de 2011]

21 La Región, 03.03.2011,< http://www.laregion.es/noticia/145208/chaves/verin/fuerzas/contra/telepeaje>, [consultado el 7 de marzo de2011]

22 Ver <http://es.eurocidadechavesverin.eu/_cf/29988 > [consultado el 21 de marzo de 2011] 
dades estatales, conformaría y confirmaría horizontes político-territoriales transfronterizos e interestatales lejos de la «necesaria colaboración entre Lisboa y Madrid» ${ }^{23}$ para la profundización de los objetivos del proyecto.

Sería a través de esa dotación de personalidad jurídica para operar desde un horizonte europeo donde la regionalización transfronteriza se convierte en un referente con interlocución propia y reconocida; además del reconocimiento a través de la dotación financiera, la Eurociudad Chaves-Verín ha estado presente como proyecto de referencia en los llamados Open Days ${ }^{24}$ de Bruselas, desde su edición de 2008. La presentación del proyecto convierte a la Eurociudad en interlocutor en la Unión Europea, de manera independiente a la de los Estados a los que pertenecen las ciudades que la integran, y como destinatarios de fondos europeos que significan el proyecto.

En cualquier caso, la consideración de un marco territorial transfronterizo en las prácticas y discursos de actores institucionales mostraría un cambio en los patrones de socialización espacial; los horizontes de referencia ya no son exclusivamente los marcados por las geografías oficiales de ambos estados; en esas geografías, y a la hora de implementar políticas, surgen otras escalas geográficas que denotan la importancia de las intervenciones de diferentes esferas políticas a la hora de conformar identificaciones socio-territoriales y regímenes de movilidades transfronterizas.

\section{REFLEXIONES FINALES}

En un editorial de la revista Political Geography, dedicado a la territorialidad en la Unión Europea y a la fronterización de Europa, Scott y van Houtum (2009: 271-273) reflexionan en torno las contradicciones y las consecuencias sociales de las territorialidades emergentes dentro de la UE. Los autores señalaban como significativa la «ausencia de un modelo geográfico-político único» para explicar la evolución de la UE durante la última década (Scott y van Houtum, 2009: 273; en cursiva en el original).

Sin embargo, aunque no exista un «único guión» (ibídem; entre comillas en el original) desde el que se puedan explicar todas estas territorialidades, sí podemos hablar de diferentes geografía políticas desplegadas desde y a través de las políticas de la Unión Europea. Las prácticas políticas, socioterritoriales y discursivas en torno a las fronteras son una de las claves de la performance de la europeidad de la Unión: el marco comunitario promueve una visión basada en las regiones como escenarios de gestión, y la regionalización transfronteriza se ha convertido en un laboratorio de prácticas para la reestructuración simbólica de escenarios sociopolíticos en los límites fronterizos interestatales. En ese sentido, las zonas fronterizas se localizan en el centro de los procesos de construcción de la esfera pública (Balibar, 1998, citado en Pickles, 2005: 362)

23 Entrevista al coordinador del Gabinete de Apoyo Técnico de la Eurociudad, 10 de febrero de 2011.

24 Los Open Days, o la Semana de las Regiones y las Ciudades, son reuniones anuales en las que las Regiones y las Ciudades de la Unión Europea mostrarían su capacidad de implementación de las políticas comunitarias, a través de su participación en debates, exposiciones y talleres en los que se trabajaría sobre las experiencias locales y su importancia para el desarrollo de la Europa de las Regiones. Ver más en http://ec.europa.eu/regional_policy/ conferences/od2011/index.cfm?nmenu=1\&sub=100, [consultado el 7 de marzo de 2011] 
La Eurociudad Chaves-Verín es uno de esos laboratorios desde los que observar las dimensiones de las prácticas de fronterización, desfronterización y refronterización en la conformación de esas geografías políticas europeas. Las intervenciones en una periferia fronteriza la convierten en centro, desde el cual se reconstruyen imaginaciones geográficas transfronterizas a través de procesos cotidianos de socialización espacial: los paquetes turísticos de la Eurociudad proponen un solo destino en dos países, delimitando el área fronteriza en torno a un río como icono geográfico; el turismo transfronterizo cambia el sentido de los límites, reconstruyendo la zona ubicada en torno al límite fronterizo como destino final; los municipios de Chaves y Verín se proyectan como el barrio de Arriba y el barrio de Abajo de la conurbación transfronteriza; el límite fronterizo, como dispositivo visual, se resignifica a través de una rehabilitación de los edificios aduaneros que lo coloca en el centro de un espacio proyectivo e institucionalmente significativo. Verín se coloca en el mapa europeo «de la mano de la ciudad lusa [Chaves] como modelo de una sociedad sin fronteras» ${ }^{25}$, como símbolo de la «construcción de la nueva Europa en un rincón periférico europeo» ${ }^{26}$. De algún modo, las prácticas de refronterización ligadas a la Eurociudad recrean distinciones entre grupos sociales en función de su grado de europeidad tal y como se practica desde la Unión Europea (Scott y van Houtum, 2009: 271).

Una mirada a la gestión de las acciones de la Eurociudad respecto a los gobiernos centrales muestra que las paradojas de estos procesos forman parte de una lectura del laboratorio en términos de dinámicas de refronterización en un contexto de desfronterización de la frontera hispano-portuguesa. Así, en relación a la ya mencionada instalación de cabinas de peaje en la autovía que enlaza Verín y Chaves y a la recogida de firmas para su eliminación, aparecen argumentos que muestran las intersecciones del proyecto con su emplazamiento en un área marginal. La recogida de firmas en contra del peaje no contó con el apoyo del sector comercial de Feces de Abaixo, ya que el desvío por la carretera nacional que realizarían los vehículos con el objetivo de evitar las tasas beneficiaría al comercio localizado en el antiguo paso fronterizo ${ }^{27}$. A unos cien metros de Feces de Abaixo, en el lado portugués del paso fronterizo, en la freguesía de Vilaverde da Raia, los edificios de aduanas eran y son de propiedad privada; se encuentran en estado de abandono y deterioro muy visibles, una vez que no han sido integrados como parte de los dispositivos visuales de la frontera proyectada desde la Eurociudad. Los carteles de «Se Vende» y «Se Alquila» en todas las dependencias son una muestra de cómo el límite fronterizo está en constante transformación.

El acceso desigual a las experiencias transfronterizas marcadas por el entorno de la Eurociudad reproduciría diferenciaciones estructuralmente ligadas a áreas rurales y a límites previamente constituidos y vividos. La participación de las zonas rurales del municipio de Verín en el proyecto es desigual, no sólo en términos de contacto de los flujos fronterizos con las actividades económicas de estas, sino también por otras razones estructurales. Así, el envejecimiento poblacional impide una presencia cualificada en los torneos interparroquiales de

25 Declaraciones del alcalde de Verín, La Región, 22.06.2009, <http://www.laregion.es/noticia. php?id=94803 ?visita $=1>$ [consultado el 23 de marzo de 2011]

26 Declaraciones del presidente de la Deputación de Ourense, Agencia EFE, 16.07.2008, <http://www.soitu. es/soitu/2008/07/16/info/1216218139_162410.html> [consultado 3 de marzo de 2010]. En cursiva en el original.

27 La Región, 03.03.2011, <http://www.laregion.es/noticia/145208/chaves/verin/fuerzas/contra/telepeaje> [consultado el 7 de marzo de 2011] 


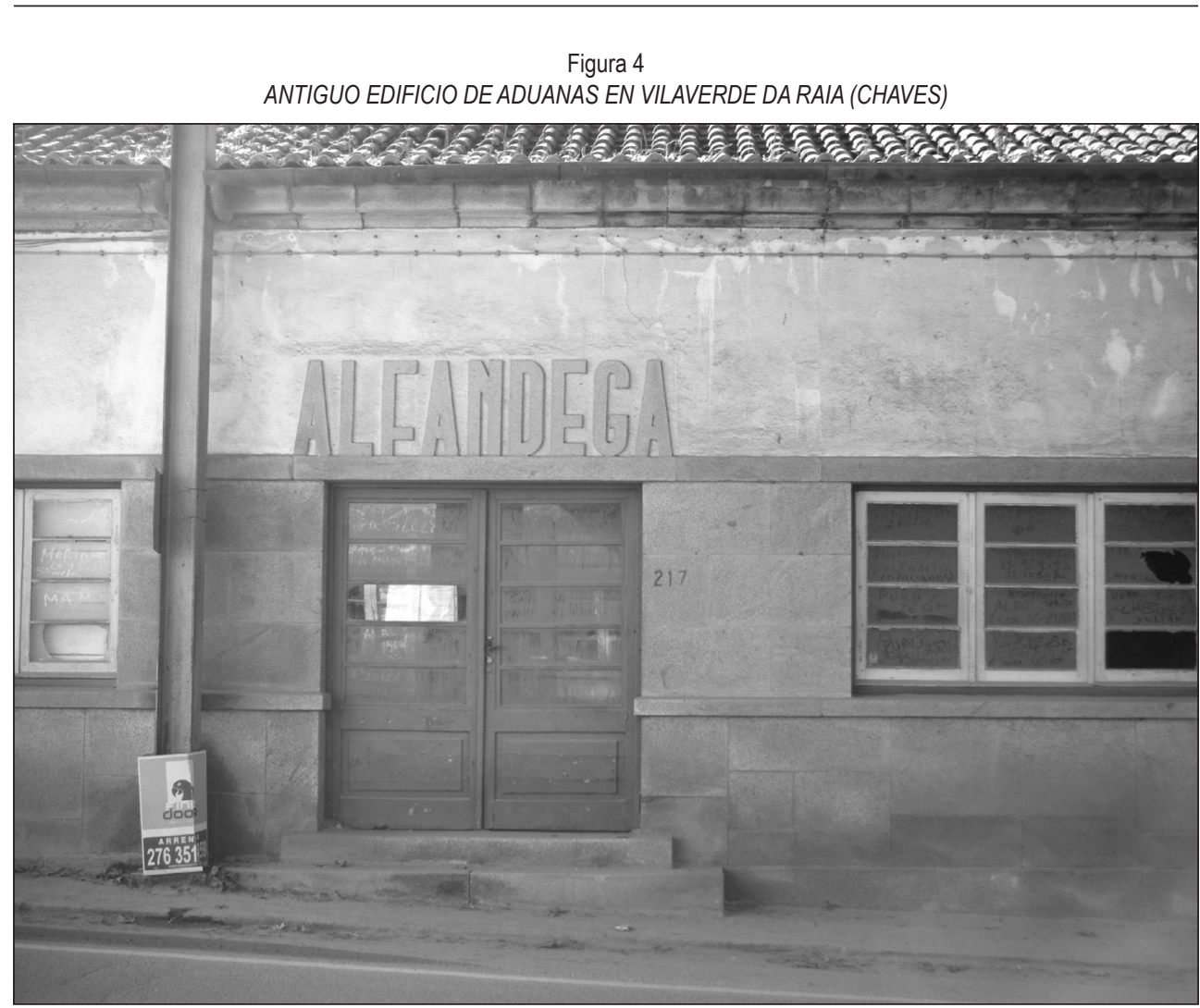

Fotografiada por la autora el 10 de febrero de 2011.

Fútbol, una de las pocas actividades que implicaría a las geografías municipales al completo, y no sólo a los núcleos urbanos.

Igualmente, la patrimonialización del Contrabando y el impulso a una narrativa hegemónica en torno a las Rutas del Contrabando cuyo recorrido impulsa la Eurociudad como actividad de esparcimiento es algo más que una experiencia para grupos de visitantes urbanos. En el caso de Vilardevós, municipio con 23 kilómetros fronterizos contiguo a Verín, estas narrativas son todavía un recurso local, difícilmente capitalizable, y relativamente alejado de la contrarrepresentación local de la frontera. La conmemoración de la transgresión simbolizada por el contrabando, e institucionalmente esponsorizada por la UE, está en constante negociación con otras historias de la frontera, y provoca diferencias (respecto a la narración) e indiferencias (respecto al contrabando como artefacto turístico) en las comunidades locales, reconstruidas para el turismo desde una perspectiva exenta de conflicto ${ }^{28}$. La recreación de memorias de subvención de los límites estatales convive con memorias locales

28 El trabajo de Ribeiro y Marques (2002), aunque circunscrito al área portuguesa, señala estas contradicciones para enfatizar la necesidad de un mayor trabajo empírico en torno al impacto de las políticas turísticas de la UE en periferias rurales desfavorecidas. 
de cooperación transfronteriza no institucionalizada, alejadas de los circuitos de producción de narrativas turísticas. En definitiva, se reproducen otros límites, que profundizarían en la periferialización de las áreas rurales excluidas del ámbito de acción de la Eurociudad.

Los significados de la frontera en esta área, entonces, están ligados tanto a su concepción como barrera como a su representación como lugar de contacto, y a una cotidiana negociación de narrativas que reconfigura los referentes de socialización espacial usualmente vinculados con el límite fronterizo, que, en este caso, se mostraría como un proceso paradójico, y en constante reconstrucción.

\section{AGRADECIMIENTOS}

Este artículo forma parte de los resultados del proyecto «El discurso geopolítico de las fronteras en la construcción de la identidades nacionales: el caso de la frontera hispano portuguesa en los siglos XIX y XX», financiado por el Ministerio de Ciencia e Innovación a través del Plan Nacional de I+D (SEJ-200766159). La autora desea agradecer a los evaluadores anónimos sus comentarios y sugerencias, que han sido fundamentales para la versión final de este trabajo.

\section{BIBLIOGRAFÍA}

AGNEW, J. (2008): «Borders on the mind: re-framing border thinking». Ethics \& Global Politics, 2008, 1(4), 175-191.

BALIBAR, E. (1998): «The borders of Europe» en Cosmopolitics: Thinking and Feeling beyond the Nation (Cheah, P. y Robbins, B., eds). Minneapolis, University of Minnesota Press, 216-233.

CABERO, V., CAMPESINO, A., y LÓPEZ TRIGAL, L. (1995-1996): «Knowledge of border areas. The contributions of Spanish Geographers». Boletín de la Asociación de Geógrafos Españoles, 22, 83-96.

CAIRO, H. (2001): «Territorialidad y fronteras del Estado-nación: Las condiciones de la política en un mundo fragmentado». Política y Sociedad, 36, 29-38.

EUROCIUDAD CHAVES-VERÍN (2009): Dossier del Gabinete de Apoyo Técnico a la Eurociudad. Verín, Eurociudad Chaves- Verín/Concello de Verín.

EUROCIUDAD CHAVES-VERÍN: LA EUROCIUDAD DEL AGUA (2011): Dossier del Gabinete de Apoyo Técnico a la Eurociudad.Verín. Eurociudad Chaves-Verín/Concello de Verín.

EUROPEAN COMMISSION (EC 2002): Structural policies and European territory: Cooperation without frontiers. Luxemburgo, European Social Fund and the Cohesion Fund.

EUROPEAN COMISSION (EC 2006-1083): European Regional Development Fund, the European Social Fund and the Cohesion Fund. Luxemburgo, European Social Fund and the Cohesion Fund.

GUICHARD, F., LÓPEZ TRIGAL, L. y MARROU, L. (2000): Itinerarios transfronterizos en la Península Ibérica. Zamora. Fundación Afonso Henriques.

HIERNAUX, D. y LINDÓN, A. (Dirs.) (2006): Tratado de Geografía Humana. Barcelona. Anthropos. 
JENSEN, O. B. y RICHARDSON, T. (2004): Making European Space. Mobility, power and territorial identity. London and New York. Routledge.

KAPLAN, D. y HÄKLI, J. (Eds.) (2002): Boundaries and place. European Borderlands in Geographical Context. Boston. Rowman \& Littlefield.

KNIPPENBERG, H. (2004): «The Maas-Rhine Euroregion: A Laboratory for European Integration?» Geopolitics, 9(3), 608-626.

KUUS, M. (2010): «Critical Geopolitics», en The International Studies Encyclopedia. Vol. II. (DENEMARK, R., Ed.) Chichester, Wiley-Blackwell, 683-701.

LAGENDIJK, A (2005): «Regionalization in Europe. Stories, institutions and boundaries» en B/ordering Space (Van Houtum, H. et. al., Eds.). Aldershot, Ashgate, 77-92.

LAMBREGST, B. et.al. (2008): «Effective governance for competitive regions in Europe: the difficult case of Randstad». GeoJournal, 72, 45-57.

LEHTINEN, A. (2008): «Politics of the Interface: Displacing Trans-border Relations» en Mobility and Place: Enacting Northern European Peripheries (Bærenholdt, J. y Granås, B., eds). London, Ashgate, 193-204.

LÒPEZ TRIGAL, L, LOIS GONZÁLEZ, R.C. y GUICHARD, F. (Coord.) (1997): La articulación territorial en la raya hispano-portuguesa. Zamora, Fundación Rei Afonso Henriques.

LÓPEZ TRIGAL, L. (Coord.) (2000): La frontera hispano-portuguesa: Nuevo espacio de atracción y cooperación. Zamora, Fundación Rei Afonso Henriques.

LOIS, M. (2009): «Practicar frontera: turismo, geografías locales y relaciones sociales en las periferias europeas» en Portugal e Espanha. Entre discursos de centro e práticas de frontera (Cairo, H. et. al., Coord.). Lisboa, Colibri, 199-213.

LOIS, M. (2010): «Practicing Europe in the EU borders: local geographies, tourism and spatial socialization in the Spanish-Portuguese border». Comunicación presentada en la Conferencia Anual de la Asociación de Geógrafos Americanos (AAG). Washington D.C., Abril 2010.

LOIS GONZÁLEZ, R. C, y PLAZA, J.I. (2003): «Development policies in the peripheral and marginal areas of the border between Spain and Portugal», en Policies and strategies in marginal regions: summary and evaluations (Leimbruger, W., Majoral, R y Lee, Ch., Eds.) Aldershot/Burlington. Ashgate, 114-129.

LOZANO VALENCIA, P.J. (2007): «La Eurociudad Baiona-Donostia. ¿Ejemplo o utopía en la colaboración transfronteriza?». Boletín de la Asociación de Geógrafos Españoles, 44, 325-340.

MÁRQUEZ, J. A. (1997): «Los territorios fronterizos y los ámbitos de relación. Las relaciones en el sector andaluz». Boletín de la Asociación de Geógrafos Españoles, 25, 51-64.

NEWMAN, D. (2006): «Borders and Bordering. Towards an Interdisciplinary Dialogue . European Journal of Social Theory, 9 (2), 171-186.

NEWMAN, D. (2006b): «The lines thatt continue to separate us: Borders in our borderless world.». Progress in Human Geography, 30 (2), 1-19.

NEWMAN, D. y PAASI, A. (1998): «Fences and neighbours in the post-modern world: boundary narratives in political geography». Progress in Human Geography, 22 (2), 186-207.

O'DOWD, L. (2010): «From a 'borderless world' to a 'world of borders': bringing history back in». Environment and Planning D: Society and Space, 28, 1031-1050. 
OHMAE, K. (1990): The borderless World. London, Collins.

PAASI, A. (1996): Territories, Boundaries and Consciousness. The Changing Geographies of the Finnish-Russian Border. Chichester, Wiley

PAASI, A (2005): «Generations and the 'Development' of Border Studies». Geopolitics, 10, 663-661.

PAASI, A.(2007): «Region-Building, Boundaries and Identities in a Globalizing World» Northern Encounters in Geography, Tromsae[En línea].Disponible en http://uit.no/ getfile.php?PageId=1671\&FileId=160 [consultado el 22 de marzo de 2011].

PERKMAN, M. y SUM, N.L. (2001): «Globalization, Regionalization and Cross-Border Regions: Scales, Discourses and Governance» en Globalization, Regionalization and Cross-Border Regions (Perkman, M. y Sum, N-L., eds). Houndsmills, Palgrave Macmillan, 3-24.

PERKMANN, M. (2003): «Cross-border regions in Europe. Significance and drivers of regional cross-border co-operation». European Urban and Regional Studies, 10 (2), 153-171.

PICKLES, J (2005): «New cartographies' and the decolonization of European geographies». Area, 37 (4), 355-364.

PLAN OPERATIVO DE COOPERACIÓN TRANSFRONTERIZA ESPAÑA-PORTUGAL (POCTEP). (2009) Dirección General de Fondos Comunitarios (España)/Dirección General de Desarrollo Regional (Portugal).

PROKKOLA, E. (2007): «Cross-border Regionalization and Tourism Development at the Swedish-Finnish Border: 'Destination Arctic Circle». Scandinavian Journal of Hospitality and Tourism, 7 (2), 120-138.

PROKKOLA, E. (2008): «Border Narratives at Work: Theatrical Smuggling and the Politics of Commemoration.». Geopolitics, 13(4), 657-675.

PROKKOLA, E. (2008b): Making bridges, removing barriers. Cross-border cooperation and identity at the Finnish-Swedish border. Oulu, Nordia Geographical Publications.

RIBEIRO, M. y MARQUES, C. (2002): «Rural Tourism and the Development of Less Favored Areas-between Rhetoric and Practice». International Journal of Tourism Research, 4, 211-220.

SAARINEN, J. (2004): «Destinations in Change. The transformation process of tourist destination». Tourist Studies, 4 (2), 161-179.

SCOTT, J. W. (2000): «Euroregions, Governance and Transborder cooperation within the EU» en Borders, regions and people (Van der Welde, M. y Van Houtum, H., eds.). London, Pion, 116-136.

SCOTT, J.W. y VAN HOUTUM, H. (2009): «Reflections on EU territoriality and the 'bordering' of Europe». Political Geography, 28 (5), 271-273.

SMITH, J. et. al . (Eds.) (1984): Households and the world-economy. Beverly Hills, Sage.

STRÜVER, A. (2005): «Bor(der)ing stories: spaces of absence along the Dutch-German border» en B/ordering Space (Van Houtum, H. et. al., Eds). Aldershot, Ashgate, 221-237.

VAN HOUTUM , H. et. al. (eds.) (2005): B/ordering Space. Aldershot, Ashgate.

WOODWARD, K. y JONES, J.P. (2005): «On the border with Deleuze and Guattari» en B/Ordering Space (Van Houtum, H. et. al., Eds.). Aldershot, Ashgate, 234-248. 
